\title{
miR-221 increases osteosarcoma cell proliferation, invasion and migration partly through the downregulation of PTEN
}

\author{
JIANWEI ZHU ${ }^{1}$, FAN LIU ${ }^{1}$, QUANMING WU ${ }^{1}$ and XIANCHENG LIU ${ }^{2}$
}

Departments of ${ }^{1}$ Orthopedics and ${ }^{2}$ Oncology, Affiliated Hospital of Nantong University, Nantong, Jiangsu 226001, P.R. China

Received January 10, 2015; Accepted August 25, 2015

DOI: $10.3892 /$ ijmm.2015.2352

\begin{abstract}
Increasing evidence has demonstrated that microRNAs (miRNAs or miRs) are involved in cancer initiation and progression. Previous studies have indicated that miR-221 is one of the most consistently overexpressed miRNAs in multiple types of cancer. However, the role of miR-221 in osteosarcoma carcinogenesis and progression is not yet fully understood. Thus, the aim of the present study was to examine the expression of miR-221 in osteosarcoma and to determine the effects of miR-221 on the biological behavior of osteosarcoma cells. RT-qPCR revealed that the expression of miR-221 was significantly upregulated in the osteosarcoma tissues and osteosarcoma cell lines $(\mathrm{P}<0.05)$. In order to explore the role of miR-221 in osteosarcoma, the expression of miR-221 in the human osteosarcoma cell line MG-63 was upregulated or downregulated by transfection with miR-221 mimic or miR-221 inhibitor, respectively. The results from RT-qPCR revealed that we had successfully generated MG-63 cells in which miR-221 was either overexpressed or depleted. To investigate the effects of miR-221 on osteosarcoma cell proliferation, invasion and migration, a tetrazolium-based colorimetric assay, propidium iodide (PI) staining, a Transwell migration assay and a wound healing assay were used in the present study. The results revealed that the proliferation, invasion and migration ability of the MG-63 cells in which miR-221 was overexpressed was enhanced, and the proliferation, invasion and migration ability of the MG-63 cells in which miR-221 was depleted was suppressed. The correlation between miR-221 and phosphatase and tensin homolog (PTEN) expression was investigated by RT-qPCR and western blot analysis. The results revealed that the downregulation of miR-221 significantly increased the expression of PTEN, whereas the upregulation of miR-221 significantly reduced the expression of PTEN. Taken together, our results suggest that miR-221 enhances the proliferation, invasion and migration ability of osteosarcoma cells partly by suppressing PTEN.
\end{abstract}

Correspondence to: Dr Jianwei Zhu, Department of Orthopedics, Affiliated Hospital of Nantong University, 20 Xishi Road, Nantong, Jiangsu 226001, P.R. China

E-mail: zhujianwei_nt@163.com

Key words: osteosarcoma, miR-221, proliferation, invasion, migration

\section{Introduction}

Osteosarcoma is the most common non-hematological primary bone tumor in children and adolescents, and for osteocarcoma patients the prognosis remains poor, particularly when metastasis is present at the time of diagnosis (1-4). Current strategies focus on the primary tumor and have limited efficacy for metastatic osteosarcoma (5). Treating metastatic osteosarcoma remains a challenge in oncology. Therefore, broadening our understanding of the pathogenesis and biology of metastatic osteosarcoma tumors is key to improving treatment efficacy (6), and there is an urgent need for new, targeted therapies based on biomarkers (7).

MicroRNAs (miRNAs or miRs) are small, non-coding, single-stranded RNAs of $\sim 24 \mathrm{nt}$ in length that regulate gene output at the post-transcriptional level either by mRNA degradation or translation repression $(8,9)$. Their target genes include numerous regulators of cell proliferation, differentiation, apoptosis, development, metabolism and immunity $(10,11)$. The deregulation of miRNA expression may contribute to tumorigenesis and other pathophysiologies associated with cancer (12). miRNAs have been proven to cause the widespread disruption of various function in many types of cancer (13). Emerging evidence has indicated that miRNAs participate in human carcinogenesis as oncogenic miRNAs (increasing tumor progression) and tumor suppressor miRNAs (inhibiting tumor progression) (11,14-16).

miR-221, which is highly conserved in vertebrates, is encoded on the $\mathrm{X}$ chromosome in humans (17). A number of studies, to date, have examined the role of miR-221 in cancer development either as an oncogenic miRNA or a tumor suppressor miRNA. The overexpression of miR-221 has been observed in a several types of cancer, including glioblastoma, hepatocellular carcinoma, and gastric, breast, prostate and pancreatic cancer (17-21). Fornari et al (19) suggested that miR-221 had an oncogenic function in hepatocarcinogenesis. Garofalo et al (21) reported that miR-221 was overexpressed in aggressive hepatocarcinoma and non-small cell lung cancer cells, as compared with less invasive and/or normal liver and lung cells. The high levels of miR-221 in breast cancer results in a poorly differentiated, mesenchymal-like phenotype (22). Galardi et al (23) demonstrated that the overexpression of miR-221 was one of the factors contributing to the oncogenesis and progression of prostate carcinoma. He et al (24) demonstrated that miR-221 silencing inhibited the malignant 
properties of liver cancer in vitro and in vivo. The antagonism of miR-221 has been shown to reduce the growth of colon tumors in mice (25). Gan et al (26) demonstrated that the downregulation of miR-221 enhanced the sensitivity of breast cancer cells to tamoxifen through the upregulation of tissue inhibitor of metalloproteinases 3 (TIMP3). In view of the above, we hypothesized that miR-221 may serve as an oncogenic miRNA in multiple types of cancer. However, Felli et al (27) indicated that miR-221 inhibited normal erythropoiesis and erythroleukemic cell growth at least in part via the down-modulation of the kit receptor. In the present study, we provide evidence that miR-221 plays the role of a tumor suppressor in erythrocytes, which indicates that miRNA function is exclusively dependent on the cellular context and tumor type.

Nonetheless, the question of whether miR-221 is deregulated in osteosarcoma and its role in osteosarcoma carcinogenesis and progression remain to be elucidated. Thus, the aim of the present study was to examine miR-221 expression in osteosarcoma tissues and cells by RT-qPCR and to observe the changes in thee proliferation, invasion and migration ability of MG-63 cells following the increase and reduction in the expression of miR-221. Our data may provide important scientific information for prognosis prediction and targeted therapy for osteosarcoma.

\section{Materials and methods}

Reagents. All cell culture reagents were purchased from Gibco-BRL (Grand Island, NY, USA). The mirVana miRNA Isolation kit, SuperTaq polymerase and the mirVana qRT-PCR miRNA detection kit were purchased from Ambion (Austin, TX, USA). Osteosarcoma cell lines (MG-63 and U2OS) and a human osteoblast cell line (hFOB1.19) were obtained from the American Type Culture Collection (Rockville, MD, USA). miR-221 mimic and inhibitor were chemically synthesized by Guangzhou RiboBio Co., Ltd. (Guangzhou, China). Transfection reagents were purchased from Guangzhou RiboBio Co., Ltd. Protein extraction and quantification kits, propidium iodide (PI), dimethyl sulfoxide (DMSO) and RNase A were purchased from the Beyotime Institute of Biotechnology (Haimen, China). The ECL chemiluminescence kit was purchased from Pierce (Rockford, IL, USA). Matrigel was purchased from Collaborative Biomedical (Bedford, MA, USA). The Transwell invasion chamber was purchased from Costar (Cambridge, MA, USA). The rabbit anti-phosphatase and tensin homolog (PTEN; Cat. no. 251264) and rabbit anti- $\beta$-actin (Cat. no. 250920) polyclonal antibodies were purchased from Abbiotec LLC (San Diego, CA, USA). The horseradish peroxidase-conjugated goat anti-rabbit IgG (H+L; Cat. no. 626100) was purchased from Life Technologies (Carlsbad, CA, USA).

Specimens. Fresh osteosarcoma tissues were collected from 16 patients who had been subjected to resection surgery in our hospital (the Affiliated Hospital of Nantong University, Nantong, China) between January 2009 and July 2014. None of the patients had recevied any pre-operative treatment, and all cases were pathologically diagnosed as osteosarcoma post-operatively. Twelve fresh normal bone tissues were also obtained at this time. All tissue samples were immediately frozen in liquid nitrogen for use in subsequent experiments. The study protocol was approved by the Ethics Committee of
The Affiliated Hospital of Nantong University, Nantong, China. All patients provided written informed consent prior to the isolation of the samples.

Cell culture and transfection. The osteosarcoma cell lines (MG-63 and U2OS) were maintained in RPMI-1640 (Gibco-BRL) supplemented with $10 \%$ fetal bovine serum (FBS) and $1 \%$ penicillin-streptomycin (Gibco-BRL) at $37^{\circ} \mathrm{C}$. Human osteoblast hFOB1.19 cells were maintained in DMEM:Ham's F-12 containing 10\% FBS and geneticin (400 $\mu \mathrm{g} / \mathrm{ml}$; Gibco-BRL) at $34^{\circ} \mathrm{C}$ in an incubator with $5 \% \mathrm{CO}_{2}$.

To enforce miR-221 expression or suppress miR-221 expression in MG-63 cells, the cells were transfected with miR-221 mimic or inhibitor, and these cells then served as the overexpression group and knockdown group, respectively. MG-63 cells transfected with control miRNA were used as the control group. On the day of transfection, MG-63 cells at approximately 70-80\% confluence were moved to antibiotic-free medium. The following day, the cells were transfected with $50 \mathrm{nM}$ miR-221 mimic and $100 \mathrm{nM}$ miR-221 inhibitor using Lipofectamine ${ }^{\mathrm{TM}} 2000$ reagent (Thermo Fisher Scientific, Waltham, MA, USA), according to the manufacturer's instructions.

Reverse transcription-quantitative PCR (RT-qPCR). Total RNA was isolated from the osteosarcoma tissues and cells using the mirVana miRNA Isolation kit according to the manufacturer's instructions. Reverse transcription was performed using the TaqMan MicroRNA Reverse Transcription kit. qPCR was performed using TaqMan Universal PCR Master Mix, and TaqMan MicroRNA assay primers for human miR-221. TaqMan microRNA assays (Applied Biosystems, Foster City, CA, USA) were performed using the 7500 Fast Real-Time PCR system. The levels of miRNA were normalized to U6 controls. The cycle threshold $(\mathrm{Ct})$ values, corresponding to the PCR cycle number at which fluorescence emission reaches a threshold above baseline emission, were determined and the relative miRNA expression was calculated using the $2^{-\Delta \Delta \mathrm{Ct}}$ method, as previously described (28).

Western blot analysis. Total proteins were extracted from the osteosarcoma tissues and cells using RIPA buffer (Beyotime Institute of Biotechnology) and quantified with a BCA assay kit (Beyotime Institute of Biotechnology). Proteins were then transferred to polyvinylidene fluoride (PVDF; Amresco, Inc., Solon, OH, USA) membranes following SDS-PAGE. The PVDF membranes were blocked for $1 \mathrm{~h}$ at an ambient temperature in blocking buffer. They were then incubated with primary antibody at $4^{\circ} \mathrm{C}$ overnight in blocking buffer. The PVDF membranes were then washed in TBST for $3 \times 5 \mathrm{~min}$, and probed with corresponding secondary antibody for $2 \mathrm{~h}$ at ambient temperature. After being washed with TBST, autoradiography was conducted with ECL chemiluminescence reagents (Pierce, Rockford, IL, USA). The relative expression of the target protein was evaluated, with the gray value ratio of the target protein content being compared to the to $\beta$-actin content (target protein $/ \beta$-actin).

Cell viability and proliferation. When the population of the MG-63 cells cultured in 96-well plates reached optimal densities, we replaced the culture medium with $100 \mu$ l fresh culture 

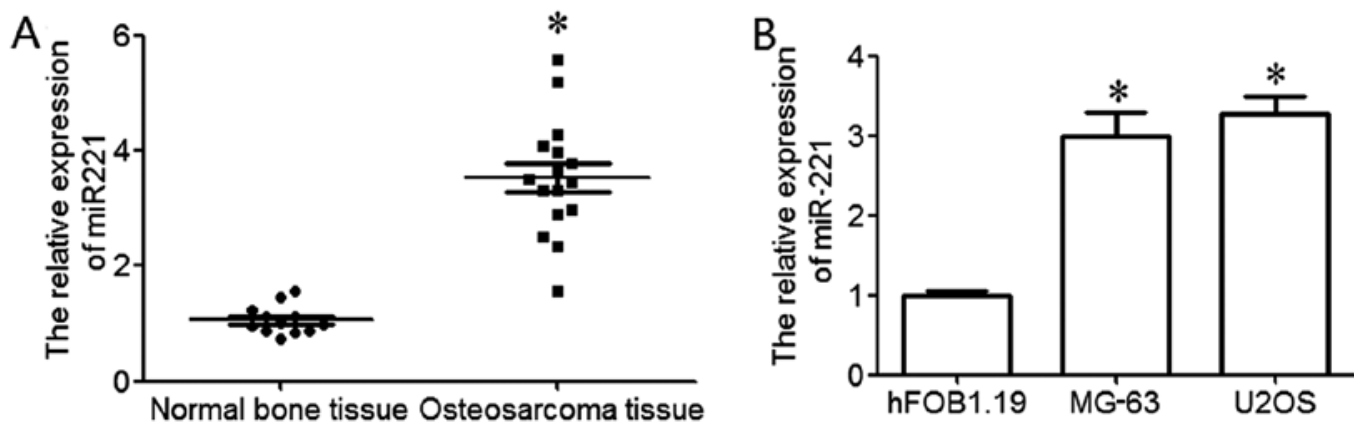

Figure 1. Relative expression of miR-221 in osteosarcoma tissues and cell lines. (A) Relative expression of miR-221 in osteosarcoma tissues compared to expression in normal tissues. ${ }^{*} \mathrm{P}<0.05$ compared to normal bone tissues. (B) Relative expression of miR-221 in osteosarcoma cell lines. ${ }^{*} \mathrm{P}<0.05$ compared to normal hFOB1.19 cells.

medium. We then added $10 \mu \mathrm{l}$ of the $12 \mathrm{mM}$ MTT stock solution to each well and incubated it for $4 \mathrm{~h}$ at $37^{\circ} \mathrm{C}$. Subsequently, we added $100 \mu \mathrm{l}$ SDS-HCl solution $(10 \mathrm{ml} 0.01 \mathrm{M} \mathrm{HCl}$ was mixed with $1 \mathrm{mg}$ ) to each well, mixed thoroughly and incubated the plates at $37^{\circ} \mathrm{C}$ for $4 \mathrm{~h}$ in a humidified chamber. Finally, we mixed each sample again using a pipette. The absorbance at $570 \mathrm{~nm}$ was measured using a plate reader, and a measurement of $690 \mathrm{~nm}$ was used as a reference. By using this procedure, only viable cells with functioning mitochondria oxidized MTT to a violet-red reaction product.

The osteosarcoma MG-63 cells were cultured in serum-free medium for $24 \mathrm{~h}$ to synchronize and then cultured in complete medium for $24 \mathrm{~h}$. The MG-63 cells were then harvested and fixed in $0.5 \mathrm{ml} 70 \%$ precooled ethanol. The fixed MG-63 cells were stored on ice for 1 day. The following day, the fixed MG-63 cells were washed and then pre-treated with RNAse (DNAsefree) $(10 \mu \mathrm{g} / \mathrm{ml})$ for $30 \mathrm{~min}$ at $37^{\circ} \mathrm{C}$. PI was then added to the solution at a final concentration of $100 \mu \mathrm{g} / \mathrm{ml}$ and incubated with the MG-63 cells for $30 \mathrm{~min}$ at room temperature in the dark. The cell cycle was evaluated by flow cytometry (BD FACSCalibur ${ }^{\mathrm{TM}}$; BD Biosciences, San Jose, CA, USA). For cell cycle analysis, data were expressed as fractions of cells in different cycle phases, and each experiment was repeated 3 times.

Wound healing assay. Cell mobility was assessed using a wound healing assay. Equal numbers of MG-63 cells from each group were seeded near confluence as a monolayer in 6-well plates. Gently and slowly, we scratched the monolayer with a sterile plastic pipette tip, across the center of the well. After scratching, we gently washed the well twice with serum-free culture medium to remove the detached cells. We then replenished the well with fresh medium. Twenty-four hours later, MG-63 cells that had migrated into the wounded area or cells with extended protrusion from the border of the wound were visualized and photographed under an inverted microscope (XDS-1B; Wuzhou New Found Instrument Co., Ltd, Guangxi, China). The distance that MG-63 cells migrated into the wounded area was measured by subtracting the distance at $24 \mathrm{~h}$ after wound healing from the initial distance. The results were based on 3 independent experiments.

Transwell invasion assay. The mechanisms involved in tumor cell invasion are very important. The most commonly used in vitro invasion assay is a modified Boyden chamber assay.
Transwell inserts with $8-\mu \mathrm{m}$ porous membranes were used for the assay. Matrigel $(20 \mu \mathrm{l})(1 \mathrm{mg} / \mathrm{ml})$ was added in order to evenly cover the surface of the Transwell insert and create the Matrigel membrane after washing the chamber with serum-free medium, which divided the chamber into upper and lower chambers. The MG-63 cells were washed 3 times with serum-free medium and added to the top chamber in serum-free medium. The bottom chamber was filled with medium containing $10 \%$ FBS, which acted as chemoattractant. The MG-63 cells were incubated for $48 \mathrm{~h}$ at $37^{\circ} \mathrm{C}$ in a $5 \% \mathrm{CO}_{2}$ humidified atmosphere. Following incubation for $48 \mathrm{~h}$, the MG-63 cells on the top surface of the chamber were removed with cotton swabs and the MG-63 cells which had invaded into the Matrigel membrane were fixed with $4 \%$ formaldehyde for $15 \mathrm{~min}$, stained with a crystal violet solution for $10 \mathrm{~min}$, visualized under a phase-contrast microscope (Leica DMIL 090-135.001; Leica Company, Wetzlar, Germany), and photographed. The results are presented as the means \pm SD, and the experiment was repeated 3 times for each group.

Statistical analysis. SPSS 17.0 software was used to analyze the data in the present study. One-way ANOVA was used to compare 3 or more groups or variables for statistical significance. A P-value $<0.05$ was considered to indicate a statistically significant difference.

\section{Results}

miR-221 is overexpressed in osteosarcoma. RT-qPCR was used to examine the expression level of miR-221 in osteosarcoma. The results revealed that miR-221 was significantly upregulated in osteosarcoma tissues compared to normal bone tissues ( $\mathrm{P}<0.05$; Fig. 1A). In order to further explore the expression of miR-221 in osteosarcoma cell lines, 2 osteosarcoma cell lines (MG-63 and U2OS) and a human osteoblastic cell line (hFOB1.192) were used in the present study. The results revealed that miR-221 expression in osteosarcoma cell lines was significantly upregulated compared to the normal human osteoblastic cell line, hFOB1.19 ( $\mathrm{P}<0.05$; Fig. 1B). These data indicate that the upregulation of miR-221 plays an important role in the development and progression of osteosarcoma.

miR-221 increases $M G-63$ cell viability. MG-63 cells were used to further examine the effects of miR-221. To enforce miR-221 expression or inhibit miR-221 expression in the MG-63 cells, 

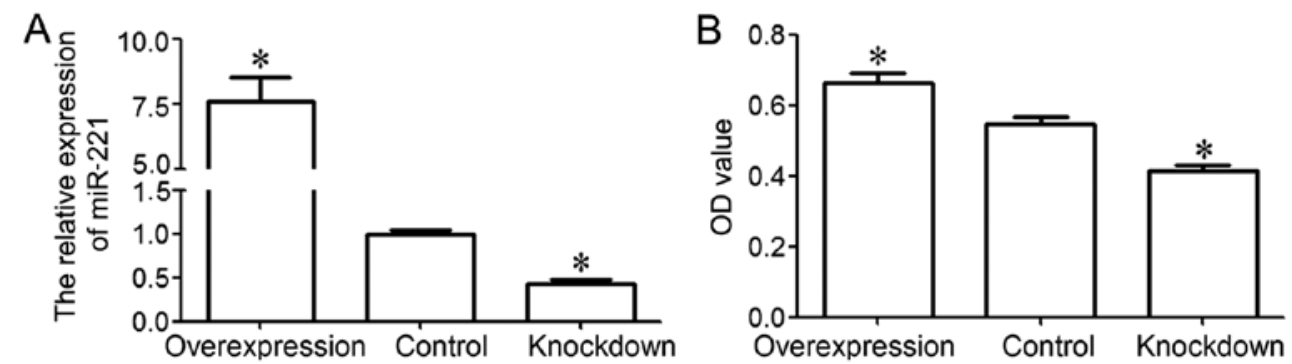

Figure 2. Expression of miR-221 in MG-63 cells and the effect of miR-221 on MG-63 cell viability. (A) Expression of miR-221 in MG-63 cells. "P<0.05 compared to control. (B) miR-221 enhances MG-63 cell viability. ${ }^{*} \mathrm{P}<0.05$ compared to control.
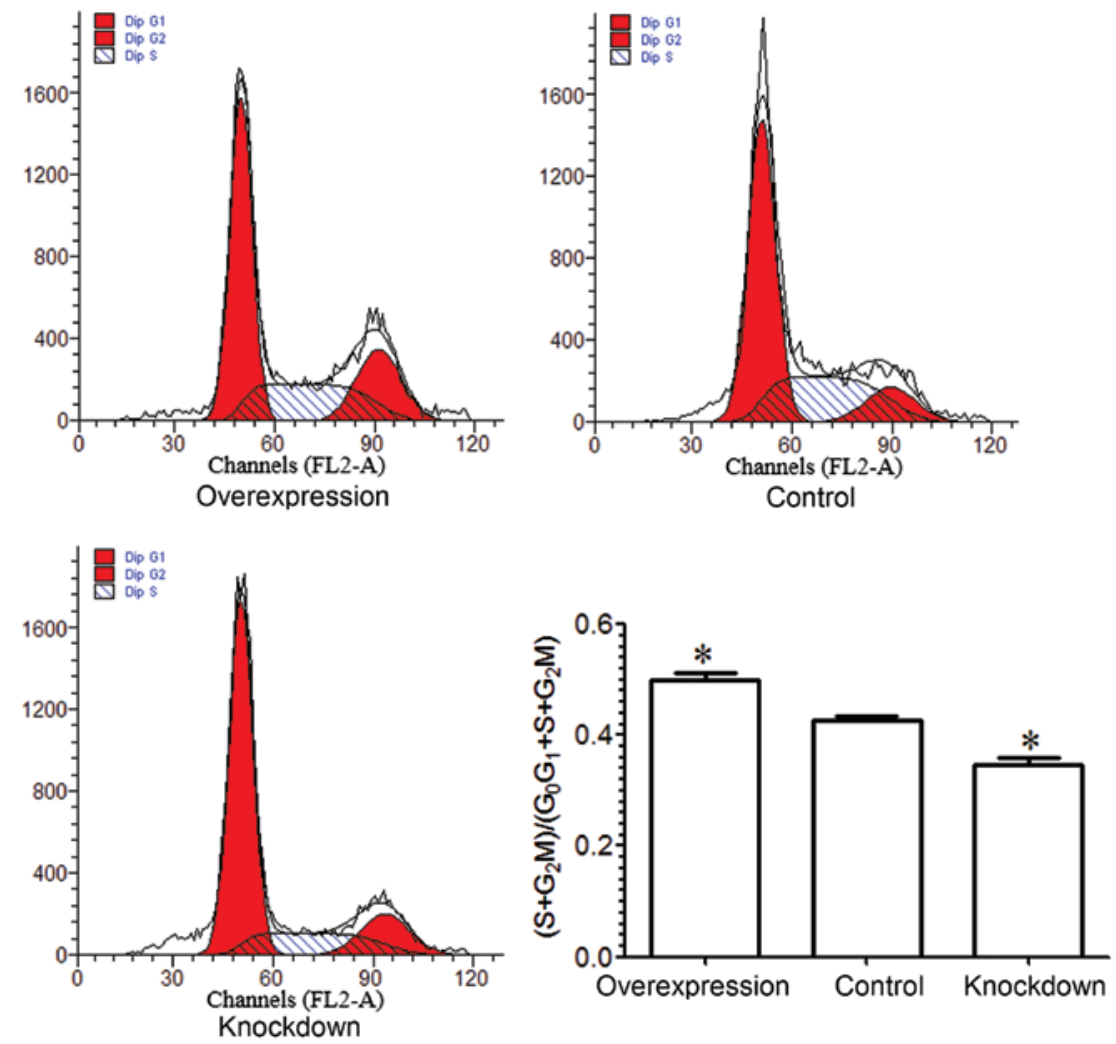

Figure 3. miR-221 enhances MG-63 cell proliferation. ${ }^{*} \mathrm{P}<0.05$ compared to control. These data were analyzed by one-way ANOVA. All experiments were repeated 3 times with 3 replicates each.

the cells were transfected with miR-221 mimic or inhibitor, and these cells then served as the overexpression group and knockdown group, respectively. The MG-63 cells transfected with scrambled oligonucleotides was used as the control group. The results from RT-qPCR revealed that miR-221 was significantly upregulated in the overexpression group and significantly downregulated in the knockdown group, as compared to the control group $(\mathrm{P}<0.05$; Fig. $2 \mathrm{~A})$. These data demonstrated that we effectively enforced or inhibited miR-221 expression in the MG-63 cells.

An MTT assay was performed to examine the viability of the MG-63 cells. The results indicated that MG-63 cell viability in the overexpression group was significantly higher than that in the control group, and that MG-63 cell viability in the knockdown group was significantly lower than that in the control group $(\mathrm{P}<0.05$; Fig. 2B). These results indicate that miR-221 is associated with the increase in MG-63 cell viability.
miR-221 accelerates MG-63 cell cycle progression. A flow cytometric assay was performed in order to examine the cell cycle of MG-63 cells. The results demonstrated that the percentage of MG-63 cells in the $S$ and $G_{2} M$ phase in the overexpression group was higher than that in the control group $(\mathrm{P}<0.05)$, and the percentage of MG- 63 cells in the $S$ and $G_{2} M$ phase in the knockdown group was lower than that in the control group $(\mathrm{P}<0.05$; Fig. 3). Based on the above results, we conclude that miR-221 enhances the proliferation of MG-63 cells.

miR-221 increases MG-63 cell invasion and migration. To determine the influence of miR-221 on the migratory potential of MG-63 cells, we examined the effects of miR-221 on cell migration using a classical scratch-wound healing assay. The MG-63 cells in the overexpression group exhibited a higher migration rate as compared to the MG-63 cells in the control group, and the MG-63 cells in the knockdown group exhibited 


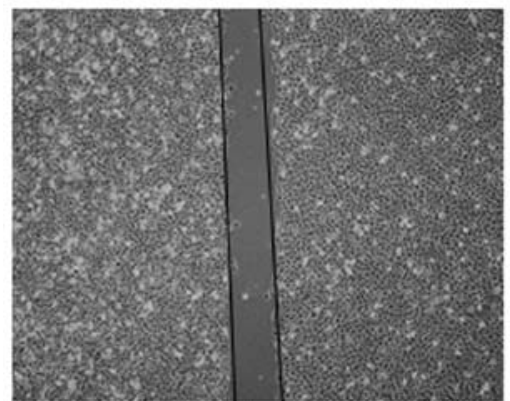

Knockdown

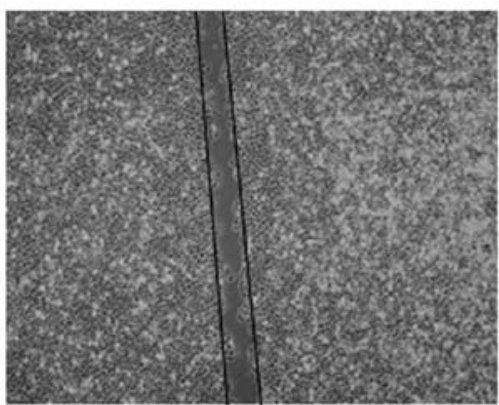

Control

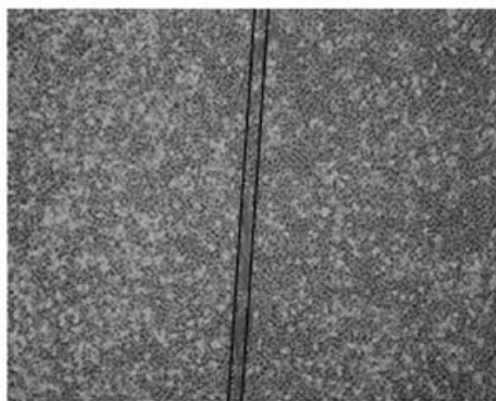

Overexpression

Figure 4. miR-221 increases MG-63 cell migration. Lines indicate the border of the healing wounds. These data were analyzed by one-way ANOVA. All experiments were repeated 3 times with 3 replicates each.

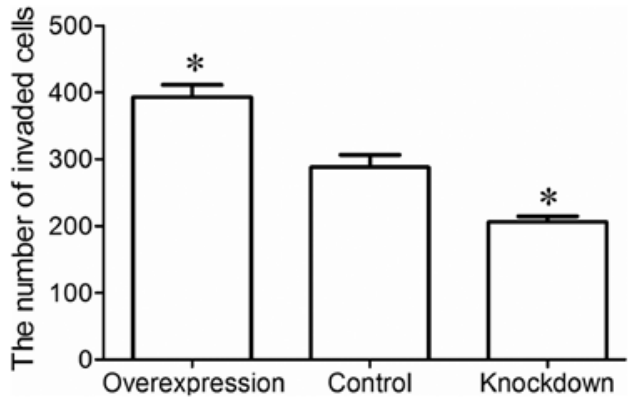

Figure 5. miR-221 increases MG-63 cell invasion. * $\mathrm{P}<0.05$ compared to control. These data were analyzed by one-way ANOVA. All experiments were repeated 3 times with 3 replicates each.
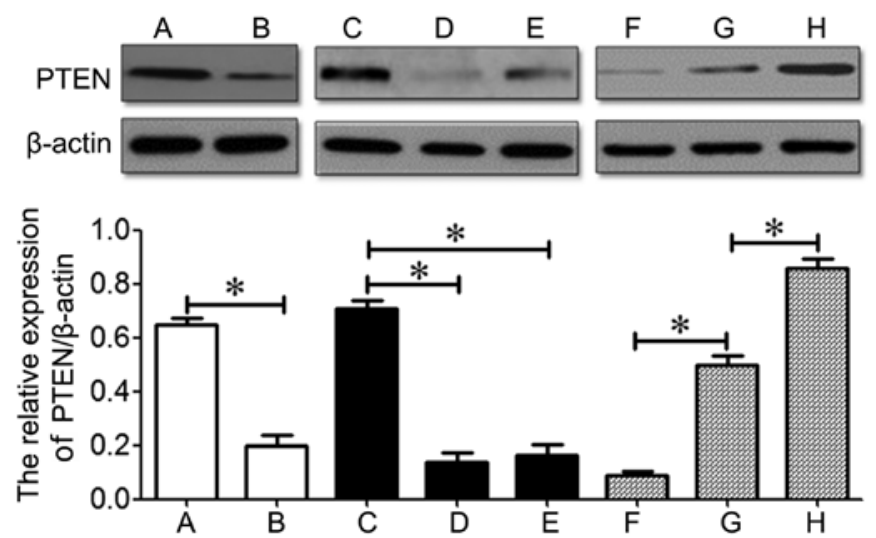

Figure 6. miR-221 suppresses the expression of phosphatase and tensin homolog (PTEN) in osteosarcoma. (A) Normal bone tissues; (B) osteosarcoma tissues; (C) hFOB1.19 cells, (D) MG-63 cells; (E) U2OS cells; (F) overexpression group; $(\mathrm{G})$ control group; $(\mathrm{H})$ knockdown group; ${ }^{*} \mathrm{P}<0.05$. These data were analyzed by one-way ANOVA. All experiments were repeated 3 times with 3 replicates each.

a lower migration rate than the MG-63 cells in the control group (Fig. 4).

Invasion assay was performed to examine the invasion ability of the MG-63 cells using a Transwell invasion chamber. The results revealed that a greater number of MG-63 cells in the overexpression group had invaded the Matrigel membrane, whereas fewer MG-63 cells in the knockdown group invaded the Matrigel membrane compared to the control group $(\mathrm{P}<0.05$;
Fig. 5). These data indicate that miR-221 enhances the invasion and metastatic ability of MG-63 osteosarcoma cells.

Effects of miR-221 on the expression of PTEN in MG-63 cells. A previous study demonstrated that miR-221 increases the invasive ability of breast cancer cells by suppressing the expression of PTEN (29). In the present study, western blot analysis revealed that PTEN was downregulated in osteosarcoma tissues and cell lines compared to the normal bone tissue or the human osteoblastic cell line, hFOB1.19 (P<0.05; Fig. 6). These data indicate the inverse correlation between miR-221 expression and PTEN expression that exists in osteosarcoma. Additionally, PTEN was downregulated in the overexpression group and upregulated in the knockdown group compared to the control group $(\mathrm{P}<0.05$; Fig. 6$)$. These results indicate that miR-221 suppresses the expression of PTEN in osteosarcoma.

\section{Discussion}

miRNAs are small non-coding RNAs that control the expression of many target mRNAs involved in a great number of normal cell functions, including differentiation, proliferation and apoptosis (30). Emerging evidence indicates that the altered expression of miRNAs is involved in cancer development and progression (31). In recent years, the dysregulation of miR-221 has been associated with a variety of human cancers, including liver and breast cancer, glioblastoma, and gastric, colon, prostate and pancreatic cancer $(17-21,24,26,32)$. It has been shown that miR-221 is significantly upregulated in liver cancer, and that it plays the role of a critical modulator in the liver cancer signaling pathway, and that miR-221 knockdown inhibits liver cancer cell proliferation, clonogenicity, migration and invasion in vitro and in vivo (24). A recent study suggested that miR-221 is responsible for resistance to tamoxifen in breast cancer (33). The downregulation of miRNA-221 increases the sensitivity of estrogen-receptor (ER)-positive MCF-7 breast cancer cells to tamoxifen (26). However, whether or not miR-221 is deregulated in osteosarcoma, and its role in osteosarcoma carcinogenesis and progression remains to be elucidated.

In the present study, we demonstrated that miR-221 was overexpressed in osteosarcoma tissues and cell lines compared to normal bone tissues and the non-cancerous osteoblastic cell line, hFOB1.19. The expression model of miR-221 in osteosarcoma in this study was consistent with that in other types 
of cancer, such as liver, breast and colon cancer $(24,26,33)$. Yang et al (34) demonstrated that miR-221 was highly expressed in PC-3 cells, and miR-221 inhibition led to reduced cell proliferation and migration in prostate cancer cells. miR-221 is frequently overexpressed in breast cancer and is associated with increased malignancy (35). It has been previously demonstrated that miR-221 knockdown induces cell apoptosis, inhibits cell cycle progression, cell proliferation, cell migration and cell invasion in breast cancer (36). Therefore, we hypothesized that miR-221 plays a positive regulatory role in tumor cell proliferation, migration and invasion. In order to determine the effects of miR-221 on osteosarcoma cells, we examined whether miR-221 was overexpressed or suppressed in MG-63 cells.

MTT assay demonstrated that the viability of MG-63 cells was significantly enhanced in the overexpression group and significantly inhibited in the knockdown group compared to the control group. Transfection with miR-221 mimic increased cell viability and miR-221 inhibitor significantly inhibited cell growth, as has also been previously demonstrated (37). These data indicate that miR-221 plays a key role in the viability of MG-63 cells. A cell cycle assay was also performed, and the results indicated that miR-221 increased MG-63 cell proliferation. Yang et al (34) found that miR-221 inhibition led to reduced cell proliferation in prostate cancer cells. It has also been shown that miR-221 targets SUN2 directly, increasing cell proliferation and tumor malignancy in medulloblastoma in vitro and in vivo (38). Sarkar et al (39) found that miR-221 inhibition upregulated the expression of PTEN and $\mathrm{p} 27^{\mathrm{kip} 1}$, and led to the inhibition of the proliferation of MiaPaCa-2 and Panc- 1 cells. The above results suggest that miR-221 promotes the growth and proliferation of osteosarcoma cells.

Increasing evidence has demonstrated that miR-221 plays an important role in the process of tumor metastasis and cell invasion $(29,40,41)$. In the present study, we investigated the effects of miR-221 on the invasion and metastasic ability of osteosarcoma cells in vitro. The scratch-wound healing assay demonstrated that miR-221 contributed to the migration of MG-63 cells, and that miR-221 knockdown inhibited the migration of MG-63 cells. The invasion assay demonstrated that miR-221 enhanced the invasive potential of MG- 63 cells, and that miR-221 knockdown suppressed the invasive potential of MG-63 cells. These data indicate that miR-221 increases MG-63 cell invasiveness and metastasis, which was consistent with findings in relation to other types of cancer. miR-221 has been shown to be significantly upregulated in metastatic minimally invasive follicular thyroid carcinoma, and some cases show a poor prognosis due to severe distant metastasis (42). Ye et al (29) demonstrated that miR-221 promoted the metastasis of human epidermal growth factor receptor 2 (HER2)-positive breast cancers by targeting PTEN. miR-221 has been proven to enhance cell survival and to induce TNF-related apoptosis-inducing ligand resistance in non-small cell lung cancer (NSCLC) cell lines through the downregulation of PTEN (43). Previous studies have demonstrated that miR-221 enhances cellular migration by suppressing PTEN $(21,44)$. Therefore, we speculated that miR-221 plays an important role in osteosarcoma, possibly by suppressing PTEN. Thus, the expression of PTEN was examined in this study. Our results indicated that PTEN was downregulated in osteosarcoma tissues and cell lines, which indicated that there was an inverse correlation between miR-221 expression and PTEN expression in osteosarcoma. The expression of PTEN was inhibited by miR-221 in the overexpression group and was improved in the miR-221 knockdown group, which indicated that miR-221 suppresses the expression of PTEN in osteosarcoma. Based on these data, we suggest that miR-221 contributes to osteosarcoma cell proliferation, invasion and migration partly through the downregulation of PTEN.

Our data demonstrated that miR-221 was significantly upregulated in osteosarcoma tissues and cell lines, suggesting that increased miR-221 expresion plays an important role in osteosarcoma oncogenesis and progression. The suppression or overexpression of miR-221 expression in MG-63 osteosarcoma cells was achieved through transfection with miR-221 inhibitor or mimic, respectively. miR-221 overexpression increased the proliferative ability of MG-63 cells, as well as invasion and migration, and miR-221 knockdown suppressed the proliferative ability of MG-63 cells, as well as invasion and migration. The downregulation of miR-221 increased the expression of PTEN, and the upregulation of miR-221 decreased the expression of PTEN. Taken together, our results suggest that miR-221 facilitates the proliferation, invasion and migration of osteosarcoma cells, at least, partly by suppressing PTEN.

\section{Acknowledgements}

The present study was supported by the Natural Science Foundation of Jiangsu Province (grant no. BK20131199) and by the fifty-fifth batch of the China Postdoctoral Science Foundation (no. 2014M551640).

\section{References}

1. Endo-Munoz L, Evdokiou A and Saunders NA: The role of osteoclasts and tumour-associated macrophages in osteosarcoma metastasis. Biochim Biophys Acta 1826: 434-442, 2012.

2. Lamora A, Talbot J, Bougras G, Amiaud J, Leduc M, Chesneau J, Taurelle J, Stresing V, Le Deley MC, Heymann MF, et al: Overexpression of smad7 blocks primary tumor growth and lung metastasis development in osteosarcoma. Clin Cancer Res 20: 5097-5112, 2014.

3. Harting MT and Blakely ML: Management of osteosarcoma pulmonary metastases. Semin Pediatr Surg 15: 25-29, 2006.

4. Jia S and Li B: Osteosarcoma of the jaws: case report on synchronous multicentric osteosarcomas. J Clin Diagn Res 8: ZD01-ZD03, 2014

5. He JP, Hao Y, Wang XL, Yang XJ, Shao JF, Guo FJ and Feng JX: Review of the molecular pathogenesis of osteosarcoma. Asian Pac J Cancer Prev 15: 5967-5976, 2014.

6. Poletajew S, Fus L and Wasiutyński A: Current concepts on pathogenesis and biology of metastatic osteosarcoma tumors. Ortop Traumatol Rehabil 13: 537-545, 2011.

7. Egas-Bejar D, Anderson PM, Agarwal R, Corrales-Medina F, Devarajan E, Huh WW, Brown RE and Subbiah V: Theranostic profiling for actionable aberrations in advanced high risk osteosarcoma with aggressive biology breveals high molecular diversity: the human fingerprint hypothesis. Oncoscience 1: 167-179, 2014.

8. Wu CW, Cheng YW, Hsu NY, Yeh KT, Tsai YY, Chiang CC, Wang WR and Tung JN: MiRNA-221 negatively regulated downstream $\mathrm{p} 27 \mathrm{Kip} 1$ gene expression involvement in pterygium pathogenesis. Mol Vis 20: 1048-1056, 2014.

9. Wolter JM, Kotagama K, Pierre-Bez AC, Firago $M$ and Mangone M: 3'LIFE: a functional assay to detect miRNA targets in high-throughput. Nucleic Acids Res 42: e132, 2014.

10. Gaál Z and Oláh E: MicroRNA-s and their role in malignant hematologic diseases. Orv Hetil 153: 2051-2059, 2012 (In Hungarian).

11. Lin S, Pan L, Guo S, Wu J, Jin L, Wang JC and Wang S: Prognostic role of microRNA-181a/b in hematological malignancies: a meta-analysis. PLoS One 8: e59532, 2013. 
12. De Sarkar N, Roy R, Mitra JK, Ghose S, Chakraborty A, Paul RR, Mukhopadhyay I and Roy B: a quest for miRNA biomarker: a track back approach from gingivo buccal cancer to two different types of precancers. PLoS One 9: e104839, 2014.

13. Xu C, Ping Y, Li X, Zhao H, Wang L, Fan H, Xiao Y and Li X: Prioritizing candidate disease miRNAs by integrating phenotype associations of multiple diseases with matched miRNA and mRNA expression profiles. Mol Biosyst 10: 2800-2809, 2014.

14. Ye S, Yang L, Zhao X, Song W, Wang W and Zheng S Bioinformatics method to predict two regulation mechanism: TF-miRNA-mRNA and lncRNA-miRNA-mRNA in pancreatic cancer. Cell Biochem Biophys 70: 1849-1858, 2014.

15. Kushwaha D, Ramakrishnan V, Ng K, Steed T, Nguyen T, Futalan D, Akers JC, Sarkaria J, Jiang T, Chowdhury D, et al: A genome-wide miRNA screen revealed miR-603 as a MGMT-regulating miRNA in glioblastomas. Oncotarget 5: 4026-4039, 2014.

16. Kalinowski FC, Brown RA, Ganda C, Giles KM, Epis MR, Horsham $\mathrm{J}$ and Leedman PJ: microRNA-7: a tumor suppressor miRNA with therapeutic potential. Int J Biochem Cell Biol 54: 312-317, 2014.

17. Garofalo M, Quintavalle C, Romano G, Croce CM and Condorelli G: miR221/222 in cancer: their role in tumor progression and response to therapy. Curr Mol Med 12: 27-33, 2012.

18. le Sage C, Nagel R, Egan DA, Schrier M, Mesman E, Mangiola A, Anile C, Maira G, Mercatelli N, Ciafrè SA, et al: Regulation of the $\mathrm{p} 27^{\mathrm{Kipl}}$ tumor suppressor by miR-221 and miR-222 promotes cancer cell proliferation. EMBO J 26: 3699-3708, 2007.

19. Fornari F, Gramantieri L, Ferracin M, Veronese A, Sabbioni S, Calin GA, Grazi GL, Giovannini C, Croce CM, Bolondi L and Negrini M: MiR-221 controls CDKN1C/p57 and CDKN1B/p27 expression in human hepatocellular carcinoma. Oncogene 27 : 5651-5661, 2008.

20. Miller TE, Ghoshal K, Ramaswamy B, Roy S, Datta J, Shapiro CL, Jacob S and Majumder S: MicroRNA-221/222 confers tamoxifen resistance in breast cancer by targeting $\mathrm{p} 27^{\mathrm{Kip1}}$. J Biol Chem 283 . 29897-29903, 2008

21. Garofalo M,Di Leva G, Romano G, Nuovo G, Suh SS, Ngankeu A Taccioli C, Pichiorri F, Alder H, Secchiero P, et al: miR-221\&222 regulate TRAIL resistance and enhance tumorigenicity through PTEN and TIMP3 downregulation. Cancer Cell 16: 498-509, 2009.

22. Howe EN, Cochrane DR and Richer JK: The miR-200 and miR-221/222 microRNA families: opposing effects on epithelial identity. J Mammary Gland Biol Neoplasia 17: 65-77, 2012.

23. Galardi S, Mercatelli N, Giorda E, Massalini S, Frajese GV Ciafrè SA and Farace MG: miR-221 and miR-222 expression affects the proliferation potential of human prostate carcinoma cel lines by targeting p27Kip1. J Biol Chem 282: 23716-23724, 2007.

24. He XX, Guo AY, Xu CR, Chang Y, Xiang GY, Gong J, Dan ZL, Tian DA, Liao JZ and Lin JS: Bioinformatics analysis identifies miR-221 as a core regulator in hepatocellular carcinoma and its silencing suppresses tumor properties. Oncol Rep 32: 1200-1210, 2014.

25. Liu S, Sun X, Wang M, Hou Y, Zhan Y, Jiang Y, Liu Z, Cao X, Chen P, Liu Z, et al: A microRNA miR-221- and miR-222-mediated feedback loop, via PDLIM2, maintains constitutive activation of nuclear factor kappaB and STAT3 in colorectal cancer cells. Gastroenterology 147: 847-859, 2014.

26. Gan R, Yang Y, Yang X, Zhao L, Lu J and Meng QH: Downregulation of miR-221/222 enhances sensitivity of breast cancer cells to tamoxifen through upregulation of TIMP3. Cancer Gene Ther 21: 290-296, 2014.

27. Felli N, Fontana L, Pelosi E, Botta R, Bonci D, Facchiano F, Liuzzi F, Lulli V, Morsilli O, Santoro S, et al: MicroRNAs 221 and 222 inhibit normal erythropoiesis and erythroleukemic cell growth via kit receptor down-modulation. Proc Natl Acad Sci USA 102: 18081-18086, 2005.

28. Livak KJ and Schmittgen TD: Analysis of relative gene expression data using real-time quantitative PCR and the 2(-Delta Delta C(T)) Method. Methods 25: 402-408, 2001.
29. Ye X, Bai W, Zhu H, Zhang X, Chen Y, Wang L, Yang A, Zhao J and Jia L: MiR-221 promotes trastuzumab-resistance and metastasis in HER2-positive breast cancers by targeting PTEN. BMB Rep 47: 268-273, 2014

30. De Tullio G, De Fazio V, Sgherza N, Minoia C, Serratì S, Merchionne F, Loseto G, Iacobazzi A, Rana A, Petrillo P, et al: Challenges and opportunities of microRNAs in lymphomas. Molecules 19: 14723-14781, 2014.

31. Xu Y, Huang Z and Liu Y: Reduced miR-125a-5p expression is associated with gastric carcinogenesis through the targeting of E2F3. Mol Med Rep 10: 2601-2608, 2014.

32. Tao K, Yang J, Guo Z, Hu Y, Sheng H, Gao H and Yu H: Prognostic value of miR-221-3p, miR-342-3p and miR-491-5p expression in colon cancer. Am J Transl Res 6: 391-401, 2014.

33. Wei Y, Lai X, Yu S, Chen S, Ma Y, Zhang Y, Li H, Zhu X, Yao L and Zhang J: Exosomal miR-221/222 enhances tamoxifen resistance in recipient ER-positive breast cancer cells. Breast Cancer Res Treat 147: 423-431, 2014.

34. Yang X, Yang Y, Gan R, Zhao L, Li W, Zhou H, Wang X, Lu J and Meng QH: Down-regulation of mir-221 and mir-222 restrain prostate cancer cell proliferation and migration that is partly mediated by activation of SIRT1. PLoS One 9: e98833, 2014.

35. Falkenberg N, Anastasov N, Rappl K, Braselmann H, Auer G, Walch A, Huber M, Höfig I, Schmitt M, Höfler H, et al: MiR-221/-222 differentiate prognostic groups in advanced breast cancers and influence cell invasion. Br J Cancer 109: 2714-2723, 2013.

36. Nassirpour R, Mehta PP, Baxi SM and Yin MJ: miR-221 promotes tumorigenesis in human triple negative breast cancer cells. PLoS One 8: e62170, 2013

37. Chen G, Dang YW and Luo DZ: Effect of miR-221 on the viability and apoptosis of hepatocellular carcinoma HepG2 cells. Zhonghua Gan Zang Bing Za Zhi 19: 582-587, 2011 (In Chinese).

38. Hsieh TH, Chien CL, Lee YH, Lin CI, Hsieh JY, Chao ME, Liu DJ, Chu SS, Chen W, Lin SC, et al: Downregulation of SUN2, a novel tumor suppressor, mediates miR-221/222-induced malignancy in central nervous system embryonal tumors. Carcinogenesis 35 : 2164-2174, 2014

39. Sarkar S, Dubaybo H, Ali S, Goncalves P, Kollepara SL, Sethi S, Philip PA and Li Y: Down-regulation of miR-221 inhibits proliferation of pancreatic cancer cells through up-regulation of PTEN, p27 ${ }^{\mathrm{kip} 1}$, p57 kip2, and PUMA. Am J Cancer Res 3: 465-477, 2013.

40. Hwang MS, Yu N, Stinson SY, Yue P, Newman RJ, Allan BB and Dornan D: miR-221/222 targets adiponectin receptor 1 to promote the epithelial-to-mesenchymal transition in breast cancer. PLoS One 8: e66502, 2013.

41. Sun T, Wang X, He HH, Sweeney CJ, Liu SX, Brown M, Balk S, Lee GS and Kantoff PW: MiR-221 promotes the development of androgen independence in prostate cancer cells via downregulation of HECTD2 and RAB1A. Oncogene 33: 2790-2800, 2014.

42. Jikuzono T, Kawamoto M, Yoshitake H, Kikuchi K, Akasu H Ishikawa H, Hirokawa M, Miyauchi A, Tsuchiya S, Shimizu K, and Takizawa T: The miR-221/222 cluster, miR-10b and miR-92a are highly upregulated in metastatic minimally invasive follicular thyroid carcinoma. Int J Oncol 42: 1858-1868, 2013.

43. Acunzo M, Visone R, Romano G, Veronese A, Lovat F, Palmieri D, Bottoni A, Garofalo M, Gasparini P,Condorelli G, et al: miR-130a targets MET and induces TRAIL-sensitivity in NSCLC by downregulating miR-221 and 222. Oncogene 31: 634-642, 2012.

44. Wang H, Xu C, Kong X, Li X, Kong X, Wang Y, Ding X and Yang Q: Trail resistance induces epithelial-mesenchymal transition and enhances invasiveness by suppressing PTEN via miR-221 in breast cancer. PLoS One 9: e99067, 2014. 